\title{
Size-consistent self-consistent configuration interaction from a complete active space: Excited states
}

\author{
N. Ben Amor and D. Maynau \\ Laboratoire de Physique Quantique, URA 505, Université Paul Sabatier, 118 route de Narbonne, \\ 31062 Toulouse Cedex, France \\ J. Sánchez-Marín and I. Nebot-Gil \\ Departamento de Química Física, Facultat de Ciencias Químicas, C/Dr Moliner, 50, 46100 Burjasot, \\ Valencia, Spain

\section{S. Evangelisti} \\ Dipartamento di Chimica Fisica e Inorganica, Università di Bologna, Viale Risorgimento 4, \\ I-40 136 Bologna, Italy
}

(Received 27 April 1998; accepted 12 August 1998)

\begin{abstract}
The self-consistent size consistent on a complete active space singly and doubly configuration interaction (SC) ${ }^{2}$ CAS-SDCI method is applied to excited states. The (SC) ${ }^{2}$ correction is performed on a closed shell state, and the excited states are obtained by diagonalization of the dressed matrix. A theoretical justification of the transferability of the improvement concerning the dressing state to all roots of the matrix is presented. The method is tested by three tests on the spectrum of small molecules. (C) 1998 American Institute of Physics. [S0021-9606(98)30843-0]
\end{abstract}

\section{INTRODUCTION}

Configuration interaction $(\mathrm{CI})^{1}$ and coupled cluster $(\mathrm{CC})^{2}$ are approximate methods because they must use truncated basis sets. However, the consequences of the truncation are not equivalent for $\mathrm{CI}$ and $\mathrm{CC}$ results. In the $\mathrm{CC}$ formalism, the wave function keeps its product form thanks to the exponential development, so that all nonvanishing products of a double excitation with a single or another double excitation remain in the wave function. This guarantees a correct scaling of the energy with the number of electrons and the absence of unlinked diagrams. Consequently, the CC methods are called size extensive (size consistent and separable). This is not the case for CI methods which loose the sizeextensivity property of the full CI. The most common truncation of a CI or CC expansion is after the singly and doubly (SD) excited configurations with respect to a single reference determinant, which corresponds to SDCI or CCSD methods.

A truncation limited to a single reference determinant is in general not sufficient for a correct description of excited states. Unfortunately, the single reference CC approach fails when the single reference determinant is not a good zeroth order function, and the generalization of $\mathrm{CC}$ to multireference problems gives rise to a lot of theoretical and practical problems. ${ }^{3,4}$ On the other hand, various methods which are more in the CI spirit have been investigated and are almost or strictly size extensive. Some of them are derived from coupled electron pair approximation (CEPA) methods ${ }^{5-8}$ and have a functional form like coupled pair functional $(\mathrm{CPF})^{9}$ or CEPA-VAR. ${ }^{10}$ Concerning multireference approaches, Laidig et al. proposed the multireference linearized quadratic coupled-cluster method (MR-LCCM), ${ }^{11,12}$ and more recently Szalay and Füsti-Molnár ${ }^{13}$ proposed a generalization called multireference averaged quadratic coupled cluster (MR-AQCC). ${ }^{14}$ Several other approximately size-extensive methods have been developed during the last few years. A very clear and accurate review is presented by Szalay and Bartlett. ${ }^{15}$

The Toulouse group has proposed the $(\mathrm{SC})^{2} \mathrm{CI}$ (sizeconsistent self-consistent $\mathrm{CI})^{16}$ method which avoids sizeextensivity errors in truncated CIs. The $(\mathrm{SC})^{2} \mathrm{CI}$ method is based on the theory of intermediate Hamiltonians. The sizeextensivity properties are obtained by adding a correction $\Delta_{i i}$ to the diagonal terms $H_{i i}$. It is said that the $\mathrm{CI}$ matrix is dressed. The dressing is commonly used on SDCI matrices, but can be applied on any type of truncated CI matrix, including an energy selected configurational space. ${ }^{16}$ Some multireference tests $\left[(\mathrm{SC})^{2} \mathrm{MRCI}\right]$ have been presented ${ }^{17,18}$ and gave encouraging results. Finally, the $(\mathrm{SC})^{2} \mathrm{CAS}-\mathrm{SDCI}$ method has been proposed with an efficient code. ${ }^{19}$ It consists in a (SC) ${ }^{2}$ treatment of a self-consistent complete active space SDCI CAS-SDCI Hamiltonian matrix. Using a CAS reference space leads to quite large CI spaces and may be costly if the number of active orbitals must be large, but the advantages are a better efficiency concerning the programmation and that a CAS space is very convenient for the study of bond breaking or excited states.

A preliminary study ${ }^{20}$ has shown that the excited eigenvalues of the dressed (SC) $)^{2}$ matrix are not phony solutions but correspond to real excited states. It is noted in Ref. 20 that, if the most relevant determinants are included in the reference space, the accuracy of the excited roots is very good. This encouraging result is obtained in spite of the fact that the dressing has a single reference nature. It is also noted in Ref. 19 in the example of the inclusion of a $\mathrm{Be}$ atom in the $\mathrm{H}_{2}$ molecule ${ }^{21}$ that when there is more than one dominant determinant in the zeroth order description of the wave function, the effect of the (SC) ${ }^{2}$ dressing depends very weakly on whether it is done with respect to one dominant determinant 
or the other. The aim of this article is to propose an extension of the (SC) $)^{2}$ CAS-SDCI method to excited states, starting from the same program. A first part gives a brief recall of the $(\mathrm{SC})^{2} \mathrm{CAS}$-SDCI method for the ground state and describes from two different points of view the modifications required to treat excited states. In this way, an analysis of the theory is provided "on the flight." It aims to understand why this method, that was conceived for the ground state, gives good excitation energies. In a second part, some test calculations are presented for chemical problems for which benchmark results can be found in the literature or have been calculated in this work.

\section{FORMALISM AND IMPLEMENTATION}

\section{A. Brief recall of the $\left[(\mathrm{SC})^{2}\right.$-CAS-SDCl] method}

The following three categories of orbitals are defined: (i) the active orbitals that define the CAS space; (ii) the inactive orbitals that are always doubly occupied in the CAS determinants; and (iii) the virtual orbitals that are always empty in the CAS determinants. The full CI space is divided into three parts:

(a) The $\mathbf{P}$ space contains all the CAS configurations and is called the reference space.

(b) The $\mathbf{Q}$ space includes all the determinants singly and doubly excited with respect to the $\mathbf{P}$ space. The union of $\mathbf{P}$ and $\mathbf{Q}$ defines the model space $\mathbf{S}$.

(c) The $\mathbf{R}$ space includes all other excitations.

The definition of the $\mathbf{Q}$ space is not unique. It may contain only the determinants that interact with one determinant of $\mathbf{P}$. This is the minimal definition. The $\mathbf{Q}$ space is maximal when it includes all the determinants with a maximum of two nonoccupied inactive orbitals (holes) and two occupied virtual orbitals (particles). These two definitions are far from being equivalent. The minimal definition presents the advantage of dealing with much smaller CI matrices, but does not give spin eigenfunction, at least if the formalism is written in determinants and not in spin configurations. The maximal definition is also the simplest to present and to program and, for the sake of simplicity, we shall take it hereafter. We will also assume that at least one closed shell determinant belongs to $\mathbf{P}$.

The wave function may be written as

$$
\psi=\psi^{P}+\psi^{Q}+\psi^{R},
$$

with

$$
\psi^{P}=\sum_{I} C_{I} \phi_{I}^{P}, \quad \psi^{Q}=\sum_{i} c_{i} \phi_{i}^{Q}, \quad \psi^{R}=\sum_{\alpha} c_{\alpha} \phi_{\alpha}^{R} .
$$

The diagonal dressing of the $\mathbf{Q}$ determinants allows the cancellation of the unlinked contributions. Their diagonal energies are shifted down under the effect of the determinants of R. The diagonal matrix element $H_{i i}$ corresponding to a given determinant $|i\rangle \in \mathbf{Q}$ is shifted by the determinant $|\alpha\rangle \in \mathbf{R}$ if a double excitation $D_{j}^{+}$is possible from $|i\rangle$ to $|\alpha\rangle$.

Note that the excitation operator $D_{j}^{+}$is defined with respect to a closed shell single reference configuration $|0\rangle .|0\rangle$ is in general the closed shell dominant determinant of the ground state but it may as well lie higher in energy than the ground state, as in the ${ }^{1} A_{1}$ state of $\mathrm{CH}_{2}$, for example. When there is no highly dominant $|0\rangle$ determinant, a multireference dressing should be the relevant solution. However, it has been shown in Ref. 19 that the results are still quite satisfactory when there are two dominant determinants and the coefficients of them are close to $2^{-1 / 2}, D_{j}^{+}$is a double excitation from the occupied to the virtual orbital of $|0\rangle$. This condition can be written as $D_{j}^{+}|0\rangle \neq 0$. Note that $D_{j}^{+}$is independent of the definition of the CAS space. $\Delta_{i i}$ may be written as

$$
\Delta_{i i}=\sum_{\substack{j, D_{j}^{+}|i\rangle \neq 0 \\ D_{j}^{+}|i\rangle \notin S}} H_{0 j} c_{j},
$$

where $\mathbf{S}=\mathbf{P}+\mathbf{Q}$ is the model space and

$$
H_{0 j}=\langle 0|H| j\rangle \text {. }
$$

Equation (3) may be rewritten as

$$
\Delta_{i i}=\sum_{j, D_{j}^{+}|i\rangle \notin S} H_{0 j} c_{j}-\sum_{j, D_{j}^{+}|i\rangle=0} H_{0 j} c_{j} .
$$

Using the concept of "excitation classes" introduced by Ruttink et al. ${ }^{22}$ and Szalay and Bartlett, ${ }^{14}$ it is possible to remember each term of Eq. (5), and this allows a rapid dressing of the CI matrix.

\section{B. Excited states of the (SC) ${ }^{2}$ dressed matrix}

In order to better understand the performance of the method for the calculation of excitation energies, we present in this section two approaches. In the first one, we present the method as a simplification of a reference-dependent but state-universal formulation of the multireference coupled cluster (MRCC) problem. In the second one, we present an approach from the quasidegenerated perturbation theory (QDPT) that allows an analysis by means of many body perturbation theory (MBPT) diagrams.

\section{The MRCC description}

The excited roots of a dressed CI matrix are very accurate ${ }^{20}$ even though the coefficients of the doubles in the ground state are used in Eq. (5). One may briefly rationalize this efficiency by noting that the dressing also applies to the excited state $\psi_{m}$ where the determinant $|i\rangle$ is now obtained from (several) references $|I\rangle,|J\rangle$ having different coefficients $C_{I}^{m}, C_{J}^{m}$.

If one refers to a Jeziorsky-Monkhorst ${ }^{23}$-type cluster expansion of the excited wave function from the references $|I\rangle$, the wave function of state $m$

$$
\psi^{m}=\sum_{I \in P} C_{I}^{m}|I\rangle+\sum_{i \notin P} c_{i}^{m}|i\rangle
$$

is written as

$$
\psi^{m}=\sum_{I \in P} e^{S_{I}} C_{I}^{m}|I\rangle
$$


Closing Eq. (7) by the left with $\langle i|$ one gets

$$
C_{i}^{m}=\left\langle i \mid \psi^{m}\right\rangle=\sum_{I \in P} C_{I}^{m}\left\langle i\left|e^{S_{I}}\right| I\right\rangle .
$$

One may define pseudocoefficients $d_{i I}$ as:

$$
d_{i I}=\left\langle i\left|\exp S_{I}\right| I\right\rangle
$$

so that

$$
c_{i}^{m}=\sum_{I} d_{i I} C_{I}^{m}
$$

Performing a double excitation $D_{k}^{+}$on $|i\rangle$, one obtains an outer space determinant $|\alpha\rangle$. A partial contribution to the coefficient of $|\alpha\rangle$ can be obtained from a summation of disconnected contributions as

$$
c_{\alpha}^{m}=\sum_{I} t_{k, I} d_{i I} C_{I}^{m}
$$

If one substitutes the reference specific diexcitation amplitude $t_{k, I}$ by the unique amplitude of the $D_{k}^{+}$double excitation for the ground state expansion from $|0\rangle$, one obtains an evaluation of $c_{\alpha}^{m}$

$$
c_{\alpha}^{m}=d_{k}^{0} \sum_{I} d_{i I} C_{I}^{m}=d_{k}^{0} c_{i}^{m} .
$$

In this equation we have used the relation (10) and we have assumed, whatever $I$ and $i$ are, that

$$
t_{k, I}=t_{k, 0}=d_{k}^{0}
$$

provided that $D_{k}^{+}$is possible on $|i\rangle$. The equalities in Eq. (13) stress the fact that $d_{k}^{0}$ depends only on $D_{k}^{+}$, the second equality being a good approximation when the coefficients of the single excitations in the ground state wave function are small since

$$
d_{k}^{0}=t_{k, 0}+\frac{1}{2} \sum_{\substack{(m, l) \\ D_{k}^{+}=M_{m}^{+} M_{l}^{+}}} t_{m, 0} t_{l, 0},
$$

where $M^{+}$is a single excitation operator and $(m, l)$ denotes the summations running over all the pairs of the single excitations satisfying the stated conditions. This derivation helps to explain the accuracy of the excited roots of the diagonally dressed $(\mathrm{SC})^{2} \mathrm{CI}$ matrix, which assumes that the amplitudes of the double excitations in the excited state may be taken as those of the ground state. However, in Eq. (12), the evaluation of $c_{\alpha}^{m}$ is rather hybrid since it combines a coefficient for the excited state $c_{i}^{m}$ with a ground state amplitude, taken from a reference determinant $|0\rangle$ which may be absent from the excited state function for symmetry reasons. More insight into the reasons for the performance of the method can be obtained by means of the QDPT.

\section{The perturbation theory description}

The $(\mathrm{SC})^{2}$ formalism does not depend on the fact that CI space is a CAS-SD. Any multireference CI (MRCI) space could be used. Only the code would be more difficult to write. In this paragraph, we shall consider that we deal with a MRCI space.
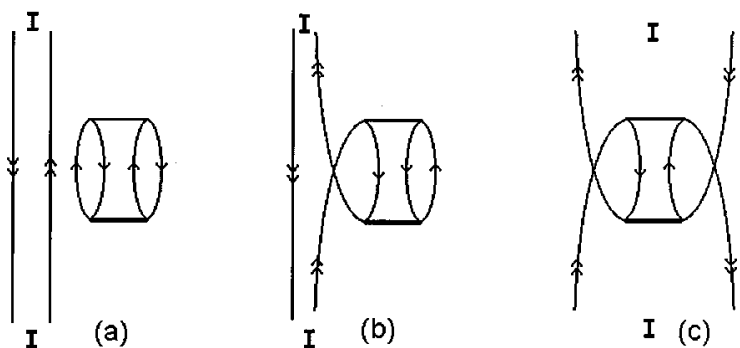

FIG. 1. Second-order-like diagrams taken into account in the diagonal $(\mathrm{SC})^{2}$ dressing of a single excitation reference. Double arrows mark the hole or particle lines belonging to the reference determinant. The thick horizontal lines mean that the coefficients of the doubles are not purely perturbative, but ground state converged $(\mathrm{SC})^{2}$ ones. The crossing lines indicate that hole or particle labels are repeated so that the diagrams violate the exclusion principle (EPV diagrams). Labels and hole-particle arrow combinations are avoided for simplicity.

It is useful to compare the (SC) ${ }^{2} \mathrm{MRCI}$ approach to an effective Hamiltonian built to second order for a few reference determinants $|I\rangle,|J\rangle, \ldots$. In this case, we can take the space $\mathbf{P}$ as the model space of the $H_{\text {eff }}$ and, in second order of QDPT we have:

$$
\left(H_{\mathrm{eff}}^{(2)}\right)_{I J}=\sum_{\alpha \notin P} \frac{\langle J|H| \alpha\rangle\langle\alpha|H| J\rangle}{E_{I}^{0}-E_{\alpha}^{0}}=\Delta_{I J} .
$$

The arguments that follow apply both to double or single excitations acting as reference determinants in P. Suppose, for simplicity, that we are in the case of excited states dominated by one single excitation $|I\rangle$ so that the $\mathbf{P}$ space includes only it (of course, the strictly degenerated spin partners are also supposed to always be included in $\mathbf{P}$ ). In this case, the index $\alpha$ in Eq. (15) runs over other single, double, and a few triple replacements, the ones that couple to $|I\rangle$. Now, we will verify whether all the second order contributions taken into account in $H_{\text {eff }}^{(2)}$ are actually included by going from SDCI to $(\mathrm{SC})^{2}$ SDCI.

We take at first the simplest (SC) ${ }^{2}$ approach to excited states, i.e., dressing the SDCI matrix of $|0\rangle$ and using, in Eq. (5), just the ground state coefficients for doubles. Even this single-reference case allows us to understand the work done by the diagonal dressing on the excited roots.

Consider the diagonal $\Delta_{I I}$ elements of Eq. (15). Each contribution due to a given $|\alpha\rangle=D_{k}^{+}|I\rangle$ has the form

$$
\frac{H_{I \alpha} \times H_{\alpha I}}{E_{I}^{0}-E_{\alpha}^{0}} \approx H_{0 k} \times \frac{H_{k 0}}{E_{0}-E_{D_{k}^{+} \Phi_{0}}}=H_{0 k} \times c_{k}^{0(1)}
$$

so that

$$
\Delta_{I I}=\sum_{\substack{D_{k}^{+} \Phi_{I} \neq 0\\}} H_{0 k} c_{k}^{0(1)} .
$$

One sees, compared to Eq. (3), that these first-order terms (and higher order contributions included in the $c_{k}^{0}$ coefficients) are actually added by the ground state (SC) ${ }^{2}$ dressing. In terms of MBPT-like diagrams, the contributions to the excited state characterized by $|I\rangle$ can be represented as shown in Fig. 1. 

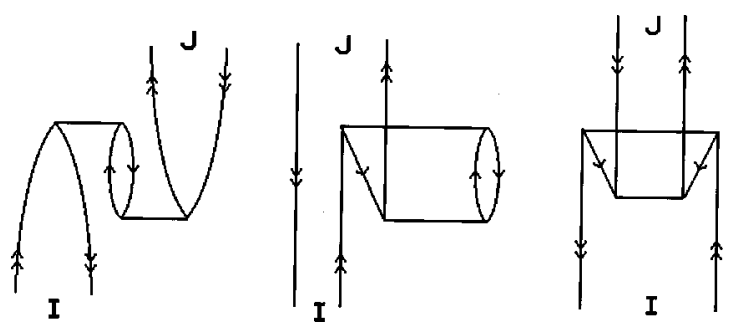

FIG. 2. Second-order-like diagrams that are not taken into account in the diagonal (SC) ${ }^{2}$ dressing of a single excitation reference.

The diagonalization of the dressed CI matrix allows the contributions (a) in Fig. 1 to remove the nonlinked terms while diagrams (b) and (c) illustrate actual contributions that remain in the energy.

Consider now the nondiagonal $\Delta_{I J}$ terms in Eq. (15). It is immediately seen that these terms are not taken into account by the (SC) ${ }^{2}$ diagonal dressing. So, very important connected processes such as those shown in Fig. 2 are missing.

We can then say that the great improvement of the excitation energies in the (SC) ${ }^{2}$ dressed SDCI comes mainly from the size-extensivity correction. This correction implies the cancellation of the nonlinked diagrams, which are diagonal in nature. However, important contributions, that can be described at the second order relative to the reference space $\mathbf{P}$, are still lacking. The natural choice, looking for an improvement, is to enlarge the CI space. One can try to include at least all the second-order effects we are comparing to the $H_{\text {eff }}$ built from a small reference. Hence, we will consider that the $(\mathrm{SC})^{2}$ dressing is added to the MR-SDCI built on this small reference made of $|O\rangle$ and a few $|I\rangle,|J\rangle \ldots$. We have now, in the model space $\mathbf{S}$, all singles, doubles, and the most relevant triples concerning the states of interest. The second-order effects described in Fig. 2 are present, as well as others that include couplings between the determinants in $\mathbf{P}$ and some triples through third-order-like diagrams such as those shown in Fig. 3. It can be remarked that higher-orderlike diagrams that involve only one triple excitation are also included in the model, as well as the nonlinked higher-order diagrams. Remember, at this point, that the diagonal sizeconsistent corrections bring into $\mathbf{S}$ the nonlinked corrections due to quadri-excitations on the doubles and pentaexcitations on the triples. Many other effects, such as some
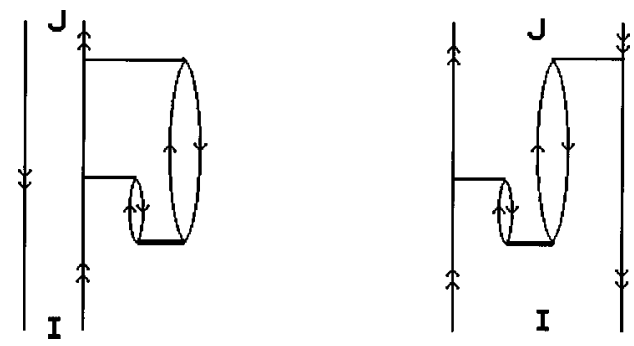

FIG. 3. Third-order-like diagrams passing through one triple that are included in MR-SDCI of a reference space made of singles after (SC) ${ }^{2}$ dressing. See the caption of Fig. 1 for further information. The EPV diagrams are not included. triple-triple interactions and exclusion principle violating (EPV) effects are in fact included in the model by means of the variational root solving.

In the case of excited states dominated by double excitations, some doubles would be included in $\mathbf{P}$. The analysis goes in the same way described above, but the most relevant quadruples, i.e., those generated by double replacements in the doubles of $\mathbf{P}$, are present in the model space $\mathbf{S}$ while the size-consistent correction implies nonlinked effects going up to some hexa-excitations.

In practice, one would be interested in keeping the $\mathbf{P}$ space as small as possible, but it must be large enough to properly characterize the state (states) of interest. However, from the point of view of computational and programming efficiency, it has been found more convenient to work with a CAS space in $\mathbf{P}$. Of course, this does not change the validity of the analysis above, which is easily generalized taking into account that all the possible excitations in the active space are present in $\mathbf{P}$. The problem in the selection of an appropriate model space is then transferred to the problem of choosing the appropriate active space of one-electron functions. All the calculations reported in this work used a CAS space in $\mathbf{P}$.

Once the dressing of the CI matrix and thus the first eigenvector has been obtained, the excited roots of the same symmetry are easy to compute, as in any other Davidson's diagonalization procedure. For the excited states of another symmetry, the process is not so straightforward. The matrix to diagonalize is a new symmetry block of the CAS-SDCI matrix, which is computed in a separate step. Is it necessary to build the new dressing operator with respect to the first state in this symmetry, as it was done for symmetry 1 ? Or can we transfer the previous dressing terms? To answer the above questions, one must consider the total CI matrix, including all $\mathbf{P}+\mathbf{Q}$ determinants of all symmetries. This is a block matrix which corresponds to the matrix we would deal with if the symmetry of the problem was not taken into account. It is possible to dress this total matrix as it is explained in Sec. II A. Note that the tables built to manage Eq. (5) are not symmetry dependent and therefore all diagonal elements can be corrected, including those of a different symmetry.

Turning back to the real procedure of treating each symmetry separately, it is now clear that it is sufficient to transfer the tables of Eq. (5) to dress each new symmetry block of the total CI matrix, which is treated as a separate matrix. This operation is performed at the beginning of the calculation, and afterwards the new symmetry dressed matrix can be diagonalized in a classical manner.

\section{NUMERICAL TESTS}

The method is tested on two examples studied in articles by Bauschlicher and Taylor ${ }^{24,25}$ and by Koch et al. ${ }^{26}$ The first example concerns $\mathrm{CH}_{2}$ for which various excited states are studied in Refs. 24, 25, and 26. The second one is the BH molecule for which the lowest excited states are given in Ref. 26. Moreover, we present a study on the $(\Delta, \Sigma)$ manifold of states of the $\mathrm{C}_{2}$ molecule for which the full $\mathrm{CI}$ calculations have been performed by one of us (S.E.). 
TABLE I. Comparison between coupled cluster and (SC) ${ }^{2}$ CAS-SDCI: the order in which the excitation energies are correct and the dependence of the computational time on the number of orbitals $n$. For the (SC) $)^{2}$ formalism, the computational time must be multiplied by the dimension of the CAS $n_{c}$. The $n^{6}$ dependence only concerns the nonactive orbitals.

\begin{tabular}{lccc}
\hline \hline & $\begin{array}{c}\text { Single } \\
\text { excitations }\end{array}$ & $\begin{array}{c}\text { Double } \\
\text { excitations }\end{array}$ & $\begin{array}{c}\text { Computational } \\
\text { time }\end{array}$ \\
\hline CC3 & 3rd order & 2nd order & $n^{7}$ \\
CCSDT-1a(MR) & 2nd order & 2nd order & $n^{7}$ \\
(SC)2CAS-SDCI & 3rd order & 3rd order & $n^{6} n_{c}$ \\
\hline \hline
\end{tabular}

Table I gives a comparison of the $\mathrm{CC} 3^{27}$ and coupled cluster of the singles, doubles and triples (linear response) $[\text { CCSDT- } 1 \mathrm{a}(\mathrm{LR})]^{28}$ methods used in Ref. 26 . This comparison concerns the order of perturbation at which the excitation energies are correct and the time dependence as a function of the number of orbitals. The order to which the excitation energies are correct depends on the degree of excitation of the determinant which is dominant in the wave function. ${ }^{26}$

\section{A. $\mathrm{CH}_{2}$ excitations energies}

The ${ }^{3} B_{1}, 1^{1} A_{1}$ and $2^{1} A_{1}$ states of $\mathrm{CH}_{2}$ are computed in Refs. 24 and 25. The carbon basis set is the Dunning double zeta contraction of the Huzinaga $(9 s 5 p)$ primitive set, ${ }^{29}$ and the hydrogen basis set is the Dunning $(4 s) /[2 s]$ contraction, ${ }^{29}$ with the exponents scaled. $2 p$ and $3 d$ polarization function are added to the hydrogen and carbon atoms. The basis sets, the geometries, and the computational details are given in Ref. 24.

The occupation of the $1^{1} A_{1}$ closed shell state and of the $1^{3} B_{1}$ and $2^{1} A_{1}$ states are

$$
\begin{aligned}
& \left(1^{1} A_{1}\right) \quad 1 a_{1}^{2} 2 a_{1}^{2} 1 b_{2}^{2} 3 a_{1}^{2} \\
& \left(1^{3} B_{1}\right) \quad 1 a_{1}^{2} 2 a_{1}^{2} 1 b_{2}^{2} 3 a_{1}^{1} 1 b_{1}^{1} \\
& \left(2^{1} A_{1}\right) \quad 1 a_{1}^{2} 2 a_{1}^{2} 1 b_{2}^{2} 1 b_{1}^{2} \text {. }
\end{aligned}
$$

In the calculations of Bauschlicher and Taylor, the $1 s$ carbon electrons are frozen and the set of active orbitals contains the $2 s$ and $2 p$ carbon orbitals and the $1 s$ of hydrogen

$$
\left(2 a_{1} 1 b_{2} 3 a_{1} 1 b_{1} 4 a_{1} 2 b_{2}\right)_{\text {CAS }}^{6} \text {. }
$$

To compare our results to those of the corresponding articles, we have kept the same conditions.
The ${ }^{3} B_{1}$ ground state is an open shell and therefore the iterative (SC) ${ }^{2}$ dressing is performed on the $1^{1} A_{1}$ first excited state. This particular condition has no consequences on the quality of the results. A comparison with the results of Bauschlicher and Taylor is given in Table II. Compared with the CAS-SDCI calculation, the (SC) ${ }^{2}$ reduces the error to the full $\mathrm{CI}$ to approximately a half.

In another study on $\mathrm{CH}_{2}$, Koch et al. performed $\mathrm{CC}$ calculations and compared their results to full $\mathrm{CI}$ and to experimental results using a correlation consistent polarized valence double zeta basis set (cc-pVDZ) spherical basis of Dunning $^{30}$ augmented with a $s$ diffuse orbital of exponent 0.015 for $\mathrm{C}$ and 0.025 for $\mathrm{H}$. This corresponds to the following basis sets: $(10 s, 4 p, 1 d) /[4 s, 2 p, 1 d]$ for the carbon atom and $(5 s, 1 p) /[3 s, 1 p]$ for $\mathrm{H}$. We have kept the same CAS space for comparison with the results of Refs. 24 and 25, in spite of the fact that the excited states investigated here-the 11 lowest singlets-are very different. As a consequence, some of them cannot be obtained by determinants built on the CAS. This allows us to test the method in a difficult situation. The excitation energies of the 11 lowest singlet states of $\mathrm{CH}_{2}$ are given in Table III with a comparison to the CC results of Ref. 26.

Table IV presents the excitation energies of $\mathrm{CH}_{2}$ with respect to the $1^{1} A_{1}$ state as a function of the single or doubled excited nature of the state. When the dominant determinant is doubly excited, the CC3 and CCSDT-1a methods fail to give correct values, since they are correct at second order only. On the contrary, the multireference character of (SC) $)^{2}$-CAS-SDCI allows us to treat any degree of excitation, provided that the dominant determinant can be built on the CAS space. If this is not the case, the method fails as occurs for the $4^{1} A_{1}$ and $3{ }^{1} B_{2}$ states. For the $4^{1} A_{1}$, the dominant determinant is singly excited, and therefore the coupled cluster approaches give accurate results. Even in this case, the (SC) ${ }^{2}$ dressing greatly improves the CAS-SDCI results. For what concerns the $2^{1} A_{2}$ state, the bad (SC) ${ }^{2}$ results are due to other reasons. The molecular orbitals are not adapted to describe it. An iterative differences dedicated CI $(\text { IDDCI })^{31}$ optimization of the orbitals by García et al. ${ }^{32}$ gave accurate results for the same level of calculation.

TABLE II. Energies of some states of $\mathrm{CH}_{2}$ at different geometries of the ground state. Comparison with the results of Refs. 24 and 25 of Bauschlicher and Taylor. Total energies in hartree. Cartesian coordinates are $\mathrm{C}(0,0,0)$ and $\mathrm{H}( \pm 1.644403,0,1.32213)$ in atomic units. $1.5 R_{e}$ means that the $\mathrm{CH}$ distance is multiplied by 1.5 and $1.70^{\circ}$ that the $\mathrm{HCH}$ angle is $170^{\circ}$. For the full CI results, the energies are in hartree. For the other methods, the values correspond to the energy differences with the full CI in mhartree.

\begin{tabular}{lccccccc}
\hline \hline & & & & $1{ }^{1} A_{1}$ & $2{ }^{1} A_{1}$ & $1{ }^{1} A_{1}$ & $2^{1} A_{1}$ \\
& $1{ }^{1} A_{1}$ & $2{ }^{1} A_{1}$ & $1{ }^{3} B_{1}$ & $\left(1.5 R_{e}\right)$ & $\left(1.5 R_{e}\right)$ & $\left(170^{\circ}\right)$ & $\left(170^{\circ}\right)$ \\
\hline Full-CI & -39.027 & -38.858 & -39.04626 & -38.89924 & -38.73570 & -38.97923 & -38.94000 \\
CASSCF & 90.6 & 95.2 & 80.3 & 75.0 & 100. & 93.7 & 92.3 \\
CAS- & 1.72 & 1.93 & 1.39 & 0.96 & 1.36 & 1.83 & 1.86 \\
$\begin{array}{l}\text { SDCI } \\
\text { CAS- }\end{array}$ & -1.58 & -2.14 & 0.00 & -1.73 & -2.31 & -1.88 & -1.80 \\
$\begin{array}{l}\text { SDCI+Q } \\
(S C)^{2}\end{array}$ & 0.78 & 1.16 & 0.81 & 0.34 & 0.80 & 1.02 & 1.04 \\
\hline \hline
\end{tabular}


TABLE III. Eleven lowest singlet states of $\mathrm{CH}_{2}$. A comparison with the values is given in Ref. 26. For the $1{ }^{1} A_{1}$ state, the values correspond to total energies. For the other states, the table gives the energy differences (vertical transitions) with the $1{ }^{1} A_{1}$ state. All energies are in hartree.

\begin{tabular}{|c|c|c|c|c|c|c|c|c|}
\hline & $\mathrm{CC} 2^{\mathrm{a}}$ & $\operatorname{CCSDLR}^{\mathrm{a}}$ & $\begin{array}{l}\text { CCSDT- } \\
1 \mathrm{a}(\mathrm{LR})^{\mathrm{a}}\end{array}$ & $\mathrm{CC}^{\mathrm{a}}$ & Full-CI ${ }^{\mathrm{a}}$ & $\begin{array}{l}\text { CAS- } \\
\text { SCF }^{b}\end{array}$ & $\begin{array}{l}\text { CAS- } \\
\text { SDCI }^{\text {b }}\end{array}$ & $(\mathrm{SC})^{2 \mathrm{~b}}$ \\
\hline $1^{1} A_{1}$ & -38.9945 & -39.0218 & -39.0249 & -39.0249 & -39.0257 & -38.9065 & -39.0209 & -39.0225 \\
\hline $2^{1} A_{1}$ & - & 0.2246 & 0.1884 & 0.1884 & 0.1711 & 0.1696 & 0.1717 & 0.1721 \\
\hline $3^{1} A_{1}$ & 0.2356 & 0.2392 & 0.2417 & 0.2392 & 0.2394 & 0.2351 & 0.2393 & 0.2394 \\
\hline $4^{1} A_{1}$ & 0.3039 & 0.3109 & 0.3139 & 0.3114 & 0.3116 & 0.5204 & 0.3454 & 0.3087 \\
\hline $1^{1} B_{1}$ & 0.2783 & 0.2835 & 0.2861 & 0.2837 & 0.2831 & 0.2658 & 0.2825 & 0.2831 \\
\hline $2^{1} B_{1}$ & - & 0.3535 & 0.3137 & 0.3136 & 0.2946 & 0.3035 & 0.2961 & 0.2962 \\
\hline $1^{1} B_{2}$ & 0.0612 & 0.0654 & 0.0680 & 0.0657 & 0.0659 & 0.0869 & 0.0683 & 0.0684 \\
\hline $2^{1} B_{2}$ & - & 0.3934 & 0.3482 & 0.3483 & 0.3273 & 0.3237 & 0.3276 & 0.3284 \\
\hline $3^{1} B_{2}$ & - & 0.4548 & 0.4102 & 0.4104 & 0.3878 & 0.4724 & 0.4266 & 0.4143 \\
\hline $1^{1} A_{2}$ & 0.2166 & 0.2153 & 0.2174 & 0.2153 & 0.2151 & 0.2216 & 0.2164 & 0.2164 \\
\hline $2{ }^{1} A_{2}$ & - & 0.4346 & 0.3888 & 0.3889 & 0.3458 & 0.3569 & 0.3685 & 0.3692 \\
\hline
\end{tabular}

${ }^{a}$ Data from Ref. 26.

${ }^{\mathrm{b}}$ This work.

\section{B. BH excitations energies}

Like $\mathrm{CH}_{2}$, the $\mathrm{BH}$ molecule is studied in Ref. 26 at full $\mathrm{CI}$ and coupled cluster levels. We have chosen the following partition of the orbitals. The $1 s$ orbital of the boron atom is inactive, and the molecular orbitals constructed with the $1 \mathrm{~s}$ of the hydrogen and the $2 s$ and $2 p$ of the boron are active

$$
(1 \sigma)_{\text {inactive }}^{2}\left(2 \sigma 3 \sigma 1 \pi_{x} 1 \pi_{y} 4 \sigma\right)_{\text {CAS }}^{4} \text {. }
$$

The details concerning the cc-pVDZ augmented basis set can be found in Ref. 26. The results are given in Table V. The $(\mathrm{SC})^{2}$ values are very close to the full CI, and improve the CAS-SDCI ones.

Concerning the nature of the states and the errors, the same remarks can be made for that of $\mathrm{CH}_{2}$. The $D{ }^{1} \Pi$, $E^{1} \Sigma^{+}$, and $G^{1} \Pi$ states do not correspond to the states obtained at a CAS level. Their dominant configurations are two single excitations from the $3 \sigma$ orbital to two different $\pi$ (for the $D^{1} \Pi$ and $G^{1} \Pi$ ) and $\sigma$ (for the $E^{1} \Sigma^{+}$) nonactive orbitals, with comparable weight on both configurations. The error for both states is about 0.02 hartree at the CAS-SDCI level, while it is only 0.004 and 0.002 for the (SC) ${ }^{2}$ results. Here again, despite the improvement due to $(\mathrm{SC})^{2}$, the CC3 results are better. On the contrary, $(\mathrm{SC})^{2}$ is more accurate when the dominant determinant is doubly excited.

TABLE IV. Excitation energies of the lowest singlet states of $\mathrm{CH}_{2}$. Energy differences between CAS-SDCI, (SC) ${ }^{2}, \mathrm{CC} 3$, and CCSDT-1a(LR). The degree of excitation of the dominant determinant of the state is given between parenthesis. All energies are in mhartree.

\begin{tabular}{lrrrc}
\hline \hline & CAS-SDCI & \multicolumn{1}{c}{$(\mathrm{SC})^{2}$} & CC3 & CCSDT-1a \\
\hline $2 A_{1}(2)$ & -0.6 & -1.0 & -17.3 & -17.3 \\
$3 A_{1}(1)$ & 0.1 & 0.0 & 0.2 & -2.3 \\
$4 A_{1}(1)$ & 33.8 & 2.9 & 0.2 & -2.3 \\
$1 B_{1}(1)$ & 0.6 & 0.0 & -0.6 & -0.3 \\
$2 B_{1}(2)$ & -1.5 & -1.6 & -19.0 & -19.1 \\
$1 B_{2}(1)$ & -2.4 & -2.5 & 0.2 & -2.1 \\
$2 B_{2}(2)$ & -0.3 & -1.1 & -21.0 & -20.9 \\
$3 B_{2}(2)$ & -38.8 & -26.5 & -22.6 & -22.4 \\
$1 A_{2}(1)$ & -1.3 & -1.3 & -0.2 & -2.3 \\
$2 A_{2}(2)$ & -22.7 & -23.4 & -43.1 & -43.0 \\
\hline \hline
\end{tabular}

\section{The $\mathrm{C}_{2}$ molecule}

The $\mathrm{C}_{2}$ molecule is investigated in the present work both at full CI and (SC) ${ }^{2}$ levels. The basis set is a $[3 s 2 p 1 d]$ contraction of the $(9 s 4 p 1 d)$ of Dunning. ${ }^{33}$ At the equilibrium distance the orbital occupation of the ground state is

$$
1 \sigma_{g}^{2} 1 \sigma_{u}^{2} 2 \sigma_{g}^{2} 2 \sigma_{u}^{2} 1 \pi_{u}^{4}
$$

The core $1 \sigma_{g}$ and $1 \sigma_{u}$ orbitals are kept frozen in all calculations. The orbitals are optimized at the CAS-SCF level with eight electrons in the eight orbitals corresponding to the $2 s$ and $2 p$ atomic orbitals

$$
\left(1 \sigma_{g} 1 \sigma_{u}\right)_{\text {frozen }}^{4}\left(2 \sigma_{g} 2 \sigma_{u} 1 \pi_{x u} 1 \pi_{y u} 3 \sigma_{g} 1 \pi_{x g} 1 \pi_{y g} 2 \sigma_{u}\right)_{\text {active }}^{8}
$$

With these orbitals, the lowest $\Sigma_{g}$ and $\Delta_{g}$ states are investigated. Most of them do not correspond to the bottom of the $\mathrm{C}_{2}$ spectrum and are quite high in energy. Two kinds of studies are presented. In the first step, the spectrum is calculated with eight electrons in eight active orbitals at the equilibrium distance and for 1.5 and 2 times the equilibrium distance. The results are given in Table VI. The $(\mathrm{SC})^{2}$ results are in very good accord with the full CI, while the dimension of the diagonalization problem has been reduced by 2 orders of magnitude. One should note that, for the 3.6 and 4.5 interatomic distances, the $\Delta_{g}$ lowest state is mostly described by four determinants and therefore no one has a weight close to one in the wave function. This state has been used to build the dressing operator. In spite of this, the mean absolute error for the (SC) $)^{2}$ excitation energies at both distances compared to full $\mathrm{CI}$ is less than $0.02 \mathrm{eV}$ ( 0.7 mhartree). In a second step, the active space is reduced for the $R e=2.40 \mathrm{bohr}$ case. The results are particularly interesting (see Table VII), since the improvement of the results due to the (SC) ${ }^{2}$ correction is very clear. For the single reference calculation, the SDCI results are very poor because a large part of the second-order contributions is lacking, while the (SC) ${ }^{2}$ results are fairly better. For larger active spaces, the case of the ${ }^{5} \Delta_{g}$ is very representative. At SDCI level, the ordering of the states is wrong, while the $(\mathrm{SC})^{2}$ dressing corrects this defect. How- 
TABLE V. Eight lowest states of BH. Comparison with the values given in Ref. 26. The energies are in $E_{H}$. For the ${ }^{1} \Sigma^{+}$ground state, the total energy is obtained by adding -25 hartree to the given values. For the other states, the table gives the energy differences with the ${ }^{1} \Sigma^{+}$state. (SC) $)^{2}$-SCF means that the orbitals are canonical SCF orbitals. The degree of excitation of the dominant determinant of the state is given between parenthesis. All energies are in hartree.

\begin{tabular}{|c|c|c|c|c|c|c|c|c|c|c|}
\hline State & $\mathrm{CC} 2^{\mathrm{a}}$ & $\begin{array}{l}\text { CCSD } \\
(\mathrm{LR})^{\mathrm{a}}\end{array}$ & $\begin{array}{c}\text { CCSDT } \\
-1 \mathrm{a}(\mathrm{LR})^{\mathrm{a}}\end{array}$ & $\mathrm{CC}^{\mathrm{a}}$ & Full-CI ${ }^{\mathrm{a}}$ & $\operatorname{Exp}^{\mathrm{b}}$ & $\begin{array}{l}\text { CAS- } \\
\text { SCF }^{c}\end{array}$ & $\begin{array}{l}\text { CAS- } \\
\text { SDCI }^{c}\end{array}$ & $(\mathrm{SC})^{2 \mathrm{c}}$ & $\begin{array}{l}(\mathrm{SC})^{2} \\
\mathrm{SCF}^{\mathrm{c}}\end{array}$ \\
\hline$X^{1} \Sigma^{+}$ & -0.1907 & -0.2176 & -0.2192 & -0.2192 & -0.2197 & - & -0.1468 & -0.2183 & -0.2192 & -0.2168 \\
\hline$A^{1} \Pi(1)$ & 0.1051 & 0.1090 & 0.1093 & 0.1085 & 0.1082 & 0.1054 & 0.1233 & 0.1088 & 0.1087 & 0.1029 \\
\hline$C^{\prime 1} \Delta(2)$ & $\ldots$ & 0.2453 & 0.2276 & 0.2275 & 0.2161 & 0.2101 & 0.2237 & 0.2214 & 0.2167 & 0.2333 \\
\hline$B^{1} \Sigma^{+}(1)$ & 0.2312 & 0.2359 & 0.2361 & 0.2350 & 0.2344 & 0.2385 & 0.2269 & 0.2345 & 0.2344 & 0.2337 \\
\hline$C^{1} \Sigma^{+}(2)$ & $\ldots$ & 0.2715 & 0.2640 & 0.2637 & 0.2571 & 0.2521 & 0.2666 & 0.2588 & 0.2575 & 0.2658 \\
\hline$D^{1} \Pi(1)$ & 0.2695 & 0.2757 & 0.2761 & 0.2749 & 0.2744 & - & - & 0.2915 & 0.2707 & 0.2733 \\
\hline$E^{1} \Sigma^{+}(1)$ & 0.2700 & 0.2849 & 0.2808 & 0.2797 & 0.2778 & 0.2819 & - & 0.2958 & 0.2759 & 0.2823 \\
\hline$G^{1} \Pi(1)$ & 0.2976 & 0.3042 & 0.3048 & 0.3034 & 0.3028 & - & 0.4298 & 0.3192 & 0.2987 & 0.3028 \\
\hline
\end{tabular}

${ }^{\mathrm{a}}$ Data from Ref. 26.

${ }^{\mathrm{b}}$ Reference 35 .

${ }^{\mathrm{c}}$ This work.

ever, the highest ${ }^{1} \Sigma_{g}^{+}$state is a very multireferential state that requires a larger CAS to be calculated with the expected accuracy (see Table VI).

\section{CONCLUSION}

The (SC) $)^{2}$ CAS-SDCI method holds both multi- and single reference aspects. The CAS-SDCI is multireference in nature, and any excited state can be described with the same accuracy as the ground state, provided that all orbitals of the determinants that dominate it are active. On the contrary, the dressing takes into account only one closed shell state, which is in general-but not necessarily, the ground state of the system. Its nature is therefore fundamentally single reference.

One could expect that such a mixture of two different approaches should lead to an improvement of the closed shell dressing state, but should deteriorate the other states and, as a consequence, the whole spectrum. It has been observed in previous and present works that this is not the case, and the reasons for the good behavior of the dressing for the excited states have been discussed. These reasons are not straightforward, and it is worth viewing some of them here in a more simple and intuitive way.

(i) The size consistency error becomes very important when the number of electrons increases. In this case, the number of inactive orbitals is large, and a great number of determinants are double excitations from the inactives to the virtuals. These double excitations are common to the closed shell state used as a dressing state and to all other states. All states will therefore benefit from an improvement in the dressing state through this treatment.

(ii) It is demonstrated that the (SC) $)^{2}$ dressing has no undesirable effect from the point of view of the QDPT. This would not be the case if state specific effects (e.g., connected triple effects) were included in the dressing and transferred to the whole set of states.

(iii) Quite often an excited state is described by more than a few determinants. It can happen that its total weight on the CAS determinants will be smaller than for the ground state, despite an accurate choice of the active orbitals. Even if we do not consider the size consistency error, it is therefore likely that the ground state will be better treated than the excited one in this case. By taking into account the determinants belonging to the $R$ external space the (SC) ${ }^{2}$ dressing plays, in this case, two different roles for the ground and the excited state. For the well described ground state, $(\mathrm{SC})^{2}$ corrects the size consistency error. For the excited state, the $(\mathrm{SC})^{2}$ dressing, which takes into account the determinants of $R$ in an indirect way, improves its description as if these determinants were added to the CI space. The improvement through the (SC) ${ }^{2}$ treatment for states that are not described by active orbitals, which can be observed in some examples in this article, has no magic or spurious origin but is due to this effect.

TABLE VI. Energies of some $\Sigma$ and $\Delta$ states of $\mathrm{C}_{2}$ at three interatomic distances. Comparison between full CI and $(\mathrm{SC})^{2}$ results for the $\Sigma$ and $\Delta$ states. For 3.6 and $4.8 \mathrm{bohr}$, the lowest state is ${ }^{1} \Delta_{g}$. For the ground state, the values correspond to absolute energies. For the other states, the values correspond to the energy differences with the lowest one. All energies are in hartree.

\begin{tabular}{|c|c|c|c|c|c|c|}
\hline & \multicolumn{2}{|c|}{$R=2.40 \mathrm{bohr}$} & \multicolumn{2}{|c|}{$R=3.60 \mathrm{bohr}$} & \multicolumn{2}{|c|}{$R=4.80 \mathrm{bohr}$} \\
\hline & full CI & $(\mathrm{SC})^{2}$ & full CI & $(\mathrm{SC})^{2}$ & full $\mathrm{CI}$ & $(\mathrm{SC})^{2}$ \\
\hline${ }^{1} \Sigma_{g}^{+}$ & -75.729938 & -75.728698 & $\ldots$ & $\ldots$ & $\ldots$ & $\ldots$ \\
\hline${ }^{1} \Delta_{g}^{g}$ & 0.0797 & 0.0815 & -75.602637 & -75.600266 & -75.536662 & -75.534133 \\
\hline${ }^{1} \Sigma_{g}^{+}$ & 0.0899 & 0.0917 & 0.0051 & 0.0050 & 0.0010 & 0.0010 \\
\hline${ }^{5} \Sigma_{g}^{+}$ & 0.1889 & 0.1895 & 0.0302 & 0.0293 & 0.0119 & 0.0114 \\
\hline${ }^{5} \Delta_{g}^{g}$ & 0.2393 & 0.2404 & 0.0976 & 0.0971 & 0.0192 & 0.0188 \\
\hline${ }^{1} \Sigma_{g}^{+}$ & 0.2586 & 0.2597 & 0.1586 & 0.1577 & 0.0397 & 0.0389 \\
\hline
\end{tabular}


TABLE VII. Energies of some $\Sigma$ and $\Delta$ states of $\mathrm{C}_{2}$ equilibrium distance. SDCI and (SC) ${ }^{2}$ results for different active spaces (single reference, four electrons in three molecular orbitals, six electrons in four molecular orbitals) compared with the full CI. The three active orbitals are the $1 \pi_{x u}, 1 \pi_{y u}$, and $3 \sigma_{g}$. When there are four active orbitals, the $2 \sigma_{u}$ is added. All energies are in hartree.

\begin{tabular}{|c|c|c|c|c|c|c|c|}
\hline & \multirow[b]{2}{*}{ Full-CI } & \multicolumn{2}{|c|}{ Single reference } & \multicolumn{2}{|c|}{$4 \mathrm{el} / 3 \mathrm{MO}$} & \multicolumn{2}{|c|}{$6 \mathrm{el} / 4 \mathrm{MO}$} \\
\hline & & SDCI & $(\mathrm{SC})^{2}$ & SDCI & $(\mathrm{SC})^{2}$ & SDCI & $(\mathrm{SC})^{2}$ \\
\hline${ }^{1} \Sigma_{g}^{+}$ & 0.0000 & 0.0000 & 0.0000 & 0.0000 & 0.0000 & 0.0000 & 0.0000 \\
\hline${ }^{1} \Delta_{g}^{g}$ & 0.0797 & 0.2000 & 0.1353 & 0.0647 & 0.0756 & 0.0719 & 0.0772 \\
\hline${ }^{1} \Sigma_{g}^{+}$ & 0.0899 & 0.2062 & 0.1363 & 0.0829 & 0.0898 & 0.0860 & 0.0905 \\
\hline${ }^{5} \Sigma_{g}^{+}$ & 0.1889 & 0.2876 & 0.2063 & 0.2661 & 0.1972 & 0.2717 & 0.1991 \\
\hline${ }^{5} \Delta_{g}^{8}$ & 0.2393 & 0.3257 & 0.2449 & 0.3019 & 0.2372 & 0.3120 & 0.2387 \\
\hline${ }^{1} \Sigma_{g}^{+}$ & 0.2586 & 0.3670 & 0.2743 & 0.3067 & 0.2316 & 0.2887 & 0.2766 \\
\hline
\end{tabular}

(iv) The (SC) ${ }^{2}$ formalism may be used in different situations. As it is presented in this article, it can be applied to systems of medium size like those that can be treated by the coupled cluster methods. For larger systems, and large basis sets, it can hardly be applied in its present form. It remains, however, an interesting approach and several implementations may be envisaged. Work is in progress to apply the method to open shell systems. Moreover one can hope that, even for large systems, the relevant information could be more efficiently concentrated on a small number of natural active orbitals, thank to methods like IDDCI.$^{31}$ Concerning the spectroscopy of large molecules, the differences dedicated CI (DDCI) method has given very encouraging results. But, for a large number of electrons, a size-consistency error should appear, and a $(\mathrm{SC})^{2}$ approach will be convenient. Finally, for large systems, a perturbative treatment may be added. The method could be compared to CASPT2 of Anderson $e t a l .{ }^{34}$ In this case, the complete active space selfconsistent field (CASSCF) step would be replaced by a CASSDCI, with a smaller number of active orbitals. With respect to second order perturbation theory with a complete active space self-consistent field reference function (CASPT2), for which the CASSCF step is size consistent, the variational step is not, and a $(\mathrm{SC})^{2}$ correction should be added.

\section{ACKNOWLEDGMENTS}

This work has been supported by the European Commission through the training and mobility research (TMR) network, Grant No. FMRX-CT 96-0079 (QUCEX). Two of us (J.S.M. and I.N.G.) acknowledge financial support from the Spanish DGCYT (Project No. P894-933). The authors are grateful to Dr. J. L. Heully and J. P. Malrieu for fruitful discussions.

${ }^{1}$ I. Shavitt, in Methods of Electronic Structure theory, edited by H. F. Schaefer (Plenum, New York, 1977), p. 189.

${ }^{2}$ F. Coester, Nucl. Phys. 1, 421 (1958); F. Coester and H. Kummel, ibid. 17, 477 (1960).

${ }^{3}$ J. Paldus, in Methods of Computational Molecular Physics, edited by S. Wilson and G. H. F. Dircksen, NATO ANSI Series, Vol. 5 (Plenum, New York, 1992), p. 99

${ }^{4}$ J. Paldus, in Relativistic and Electron Correlation Effects in Molecules and Solids, edited by G. L. Malli, NATO ANSI Series (Plenum, New York, 1994), p. 207.
${ }^{5}$ W. Meyer, Int. J. Quantum Chem., Symp. 5, 341 (1971).

${ }^{6}$ W. Meyer, J. Chem. Phys. 58, 1017 (1973).

${ }^{7} \mathrm{~W}$. Kutzelnigg, in The Method of Electronic Structure Theory, edited by H. F. Schaefer III (Plenum, New York, 1977), p. 129.

${ }^{8}$ S. Koch and W. Kutzelnigg, Theor. Chim. Acta 59, 387 (1981).

${ }^{9}$ R. Alhrichs, P. Scharf, and C. Erhardt, J. Chem. Phys. 82, 890 (1985).

${ }^{10}$ P. Pulay, Int. J. Quantum Chem., Symp. 17, 257 (1983).

${ }^{11}$ W. D. Laidig and R. J. Bartlett, Chem. Phys. Lett. 104, 424 (1984).

${ }^{12}$ W. D. Laidig, P. Saxe, and R. J. Bartlett, J. Chem. Phys. 86, 887 (1987).

${ }^{13}$ L. Füsti-Molnár and P. G. Szalay, J. Phys. Chem. 100, 6288 (1996)

${ }^{14}$ P. G. Szalay and R. J. Bartlett, Chem. Phys. Lett. 214, 481 (1993).

${ }^{15}$ P. G. Szalay and R. J. Bartlett, J. Chem. Phys. 103, 3600 (1995).

${ }^{16}$ J-P. Daudey, J-L. Heully, and J-P. Malrieu, J. Chem. Phys. 99, 1240 (1993).

${ }^{17}$ J-P. Malrieu, J-P. Daudey, and R. Caballol, J. Chem. Phys. 101, 8908 (1994).

${ }^{18}$ J. Meller, J. P. Malrieu, and J. L. Heully, Chem. Phys. Lett. 244, 440 (1995).

${ }^{19}$ N. Ben Amor and D. Maynau, Chem. Phys. Lett. 286, 211 (1998).

${ }^{20}$ J. L. Heully, J. P. Malrieu, I. Nebot-Gil, and J. Sanchez-Marin, Chem. Phys. Lett. 256, 589 (1996).

${ }^{21}$ For the $\mathrm{BeH}_{2}$ system, see also G. D. Purvis, R. Shepard, F. B. Brown, and R. J. Bartlett, Int. J. Quantum Chem. 23, 835 (1983); P. Pulay and T. P. Hamilton, J. Chem. Phys. 88, 4926 (1988).

${ }^{22}$ P. J. A. Ruttink, J. H. van Lenthe, R. Zwaans, and G. C. Groenenboom, J. Chem. Phys. 94, 7212 (1991).

${ }^{23}$ B. Jeziorski and H. J. Monkhorst, Phys. Rev. A 24, 1668 (1981).

${ }^{24}$ C. W. Bauschlicher and P. R. Taylor, J. Chem. Phys. 85, 6510 (1986).

${ }^{25}$ C. W. Bauschlicher and P. R. Taylor, J. Chem. Phys. 86, 2844 (1987).

${ }^{26}$ H. Koch, O. Christiansen, P. Jørgensen, and J. Olsen, Chem. Phys. Lett. 244, 75 (1995).

${ }^{27}$ O. Christiansen, H. Koch, and P. Jørgensen, J. Chem. Phys. 103, 7429 (1995).

${ }^{28}$ Y. S. Lee, S. A. Kucharsky, and R. Bartlett, J. Chem. Phys. 81, 5906 (1984).

${ }^{29}$ S. Huzinaga, J. Chem. Phys. 42, 1293 (1965); T. H. Dunning, ibid. 53, 2823 (1970)

${ }^{30}$ T. H. Dunning, J. Chem. Phys. 90, 1007 (1989).

${ }^{31}$ V. M. Garcia, O. Castell, R. Caballol, and J. P. Malrieu, Chem. Phys. Lett. 238, 222 (1995).

${ }^{32}$ V. M. García, M. Reguero, and R. Caballol, Theor. Chem. Acc. 98, 50 (1997).

${ }^{33}$ T. H. Dunning, Jr., J. Chem. Phys. 90, 1007 (1989).

${ }^{34}$ K. Anderson, P. A. Malmqvist, B. O. Roos, A. J. Sadlej, and K. Wolinski, J. Phys. Chem. 94, 5483 (1990); K. Anderson, P. A. Malmqvist, and Roos, J. Chem. Phys. 96, 1218 (1992).

${ }^{35}$ S. H. Bauer, G. Herzberg, and J. W. C. Johns, J. Mol. Spectrosc. 13, 256 (1964); J. W. John, F. A. Grimm, and R. F. Porter, ibid. 22, 435 (1967); K. P. Huber and G. Herzberg, Constants of Diatomic Molecules (Van Nostrand, Princeton, NJ, 1979). 